\title{
Innovations in Clinical Kidney Transplant Education by a Massive Open Online Course
}

\author{
Marlies E. J. Reinders ${ }^{1}$ • Peter G. M. de Jong ${ }^{2}$
}

Published online: 16 November 2016

(C) International Association of Medical Science Educators 2016

In January 2016, the Leiden University Medical Center (LUMC), together with the Online Learning Lab of the University of Leiden, launched the first Massive Open Online Course (MOOC) on clinical kidney, pancreas and islet transplantation (https://www.coursera.org/learn/clinicalkidney-transplantation) [1]. To follow the course, students need an advanced undergraduate or beginning graduate level with a background in biology, medicine or life sciences. It is also valuable for experienced health care providers and others interested in the research and knowledge on clinical transplantation. The course is divided into four modules: (1) Before the transplant; (2) The surgical procedures and the challenged patient, including patients with diabetes; (3) Early challenges; and (4) Late challenges after transplantation. Important questions, which are answered in the course, include:

- Which factors are of importance before renal transplantation, including knowledge on renal disease (with a special focus on diabetes), immunology, immunogenetics and patient selection of low- and high-risk patients?

- What are the options for the elderly, patients with diabetes and immunologically challenged patients?

- What are the important surgical aspects of kidney, pancreas and islet transplantation?

Marlies E. J. Reinders

m.e.j.reinders@lumc.nl

1 Department of Internal Medicine (Nephrology), Leiden University Medical Center, Albinusdreef 2, 2300 RC Leiden, The Netherlands

2 Center for Innovation in Medical Education, Leiden University Medical Center, Leiden, The Netherlands
- What are challenges in the early period after transplantation including ischemia reperfusion injury, allograft rejection and choice of optimal immune suppression?

- What are challenges in the late period after transplantation, including, infections, (skin) malignancies and cardiovascular complications and what are patient and allograft outcomes, tolerance, and novel developments?

The offered modules include a variety of different education forms including lectures, interactive patient cases, 3D animations of a kidney, pancreas and islet transplantation and of different aspects of the immune system, interviews with well-known experts in the field, and serious games to increase knowledge of the field (Fig. 1). The impact of the transplant process for the patient and the kidney donor becomes clear through interviews with patients. Electronic activities help the learner to increase knowledge about patient cases and to develop skills including clinical reasoning in an active forum. For advanced knowledge on transplantation, learners can obtain in-depth insight into specialist subjects with extra assignments.

A multidisciplinary team of professionals with different expertise is paramount in the success of transplantation. In the MOOC, these experts from our transplant team give the lectures and are responsible for the content. Therefore, the MOOC was made by a large team of professionals (13 in total). The challenge here was that all the different professionals had to be trained and that the content had to be finetuned by one and another. Learners so far value that the experts with expertise do give the lectures themselves.

The course is endorsed by various professional organizations, including the European Society of Organ Transplantation (ESOT), the Transplantation Society (TTS), the International Society of Nephrology (ISN) and the European Society of Pathology. 
Fig. 1 Screenshots taken from the Coursera platform, which demonstrate different forms of education material including lectures with talking heads (a), $3 \mathrm{D}$ animations (b), interactive patient cases (c), and interviews with experts (d) a

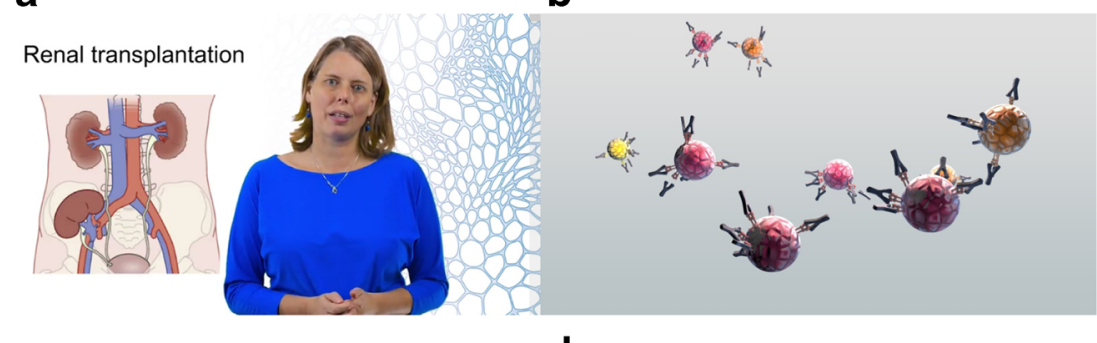

C

Patient case

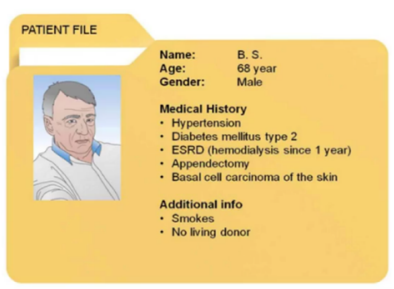

d

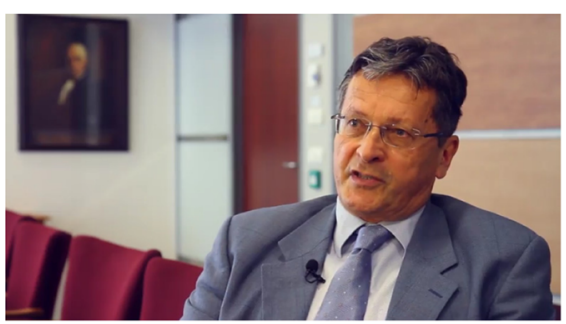

In the first 5 months, 5165 learners of all ages enrolled into the course, of which 3066 are active learners. The participants originate from over 90 countries of all continents, with the largest groups come from the United States, the Netherlands and India, and the course hereby serves a huge international and professional community of practice, which provides challenging learning opportunities. A large amount of the learners $(>70 \%)$ have a Bachelor's, Master's, Professional School or Doctorate degree, which is significantly higher than those with other MOOCs on the platform, indicating that we attract high educated professionals. The discussion platforms with patient cases in the form of electronic activities are very popular among the participants, and over 1500 posts have been placed in the first 5 months. Four moderators with a background in transplant medicine help guiding the online activities with hosting, facilitating and guiding of the learners. Currently, different steps have been made to integrate the course in campus for bachelor and master students as well as for fellows in medicine and transplant professionals. Formal studies evaluating the efficacy of the MOOCs in medicine need to be performed in the future to bring this type of education to a higher platform.

\section{References}

1. Reinders ME, de Jong PG. Innovating clinical kidney transplant education by a massive open online course. Transpl Immunol. 2016;38:1-2. 